\title{
Binarity as a tool for determining the physical properties and evolutionary aspects of A-stars
}

\author{
Mutlu Yıldız \\ Ege University, Dept. of Astronomy and Space Sciences, Bornova, 35100 Izmir, Turkey \\ email: yildiz@astronomy.sci.ege.edu.tr
}

\begin{abstract}
Double-lined-eclipsing binaries are the essential systems for the measurement of stellar masses and radii. About 50-60 systems have components (mostly A-stars) for which these values are known with an uncertainty less than $1-2 \%$. Therefore, these systems are very suitable to improve our understanding of stellar structure and evolution. In this paper, special attention is given to the assessment of the role of internal rotation of the early-type stars in selected double-lined binaries (i.e., EK Cep, PV Cas, and $\theta^{2}$ Tau): it is shown that adoption of rapidly rotating cores for such stars permits the models to be in very good agreement with the observational results including the apsidal advance rates.
\end{abstract}

Keywords. Stars: rotation, stars: interiors, stars: evolution, stars: early-type, binaries: eclipsing, stars: individual (EK Cep, PV Cas, $\theta^{2}$ Tau)

\section{Introduction}

Life, death, and the evolution of a star depend mostly on its mass. This dependence occurs since the physical conditions in the central regions of stars are primarily determined by the total mass of the overlying layers and its distribution. While outgoing radiation (luminosity) is determined by cumulative effect of the physical conditions in the central regions, the radius is entirely a function of matter-radiation and matter-matter interactions and their consequences. In addition to the mass, the comprised stellar material and physical mechanisms, which affect the stellar structure equations or macroscopic quantities, also influence the stellar structure and consequently the evolution. In this regard, the observables of a model are also functions of chemical composition, rotation and magnetic field, which have secondary roles in comparison with the stellar mass. The latter two properties are difficult to handle in model computations and are, therefore, either entirely neglected or incorporated using extremely simple forms, such as solid-body rotation, despite their importance for the early-type stars. Therefore, this study concentrates on the internal rotational properties of these stars.

The most suitable systems for the assessment of the role of the secondary effects are the stellar systems for which the masses of the components are accurately determined. These systems are double-lined eclipsing binaries (DLEBs). We have very accurate observational data of more than 100 stars in these systems. Many of these binaries show apsidal motion, which puts further constraints on the models. Uncertainties in the mass and the radius are believed to be less than 1-2\% (Andersen 1991, Harmanec 1988, Lacy et al. 2003, Popper 1980). There are many theoretical papers on the structure and the evolution of these stars (e.g., Claret \& Gimenez 1993, Lastennet \& Valls-Gabaud 2002, Pols et al. 1997, Yıldız 2003, 2004, Young et al. 2001), which mostly discussing the efficiency of the overshooting process (see Sect. 2). 
For some double-lined visual binaries, we have good quality observational data. The masses of their components determined from observations, of course, are not as accurate as those of the double-lined eclipsing binaries. In addition, for modelling, these systems have two disadvantages: 1) no direct information about the radii of the components, and 2) the problem of dividing the total brightness between the components. But, if we have several such binaries as members of an open cluster, these disadvantages can be removed. In this respect, the Hyades is a very fruitful cluster (Lebreton et al. 2001): the individual masses of the components of its five double-lined binaries are observationally known (one eclipsing and four visual binaries). Two of the visual binaries, namely, $\theta^{2}$ Tau and 51 Tau, have early-type components (Torres et al. 1997a). The primary (massive) component of $\theta^{2}$ Tau is a $\delta$ Scuti star at the top of the cluster main-sequence. Hence, it is crucial for the determination of the cluster's age. However, its model properties should be determined together with a binary which has late-type components, to limit the chemical composition and to determine its physical properties and evolutionary phase (see section $5) . \phi 342$ whose components are F-type stars is the most suitable binary system for this task (Torres et al. 1997b). F-type stars are most suitable stars for the standard modelling: Their convective zones are 1) fairly shallow which does not cause as many complications in the theoretical and the observational results, and 2) simultaneously are sufficiently deep for the effective loss of angular momentum.

A typical problem encountered in the determination of physical properties and evolutionary aspects of stars in binary systems by constructing models for the stellar interior, is due to the degeneracy in the HR diagram. That is to say, putting rotation and magnetic fields aside, at each point of the HRD, there are many different models with different chemical compositions and ages. This nonuniqueness is valid for many of the binary systems since the sensitivities of the models with different masses have very similar sensitivities to both the hydrogen and the heavy element abundances. Only in very special cases we can get (more or less) precise information concerning the structure and the evolutionary phases of the component stars: For example, binary systems with very different components (EK Cep, TY CrA, TZ Men, etc.) and systems with very small apsidal motion periods (PV Cas, GG Lup, $\zeta$ Phe, V760 Sco, etc.).

In this paper, we present and discuss results concerning the internal structure and evolutionary aspects of A- and B-type stars by constructing a rich variety of rotating models for them. The rest of the paper is organized as follows: The overshooting paradigm is discussed in Sect. 2. In Sect. 3, differential rotation is introduced for later use and the basic steps of our method for the construction of such models are presented. While model properties of the component stars in the two DLEBs (EK Cep and PV Cas) are discussed in Sect. 4 , Sect. 5 is devoted to the visual binary $\theta^{2}$ Tau of the Hyades. Finally, conclusions are drawn in Sect. 6.

\section{The overshooting paradigm}

As emphasized above, for the most part, the overshooting process is discussed in the studies on the early-type stars, especially in those of eclipsing binaries. The reason for this may be that it is easy in principle to apply it in model computations, although the phenomenon itself is surely very complex (see Roxburgh 1992) if not chaotic. The main effect of this process on the models looks essentially the same as that of a mixing process operating efficiently above the nuclear core. It is supposed that the convective cells penetrate into the radiative region and their length in this region is a small fraction of the pressure scale height $\left(H_{\mathrm{P}}\right)$ at the top of the convective core $\left(r_{\mathrm{cc}}\right)$. The adopted maximum value of this small fraction is 0.6 (Guinan et al. 2000), that is, a sphere with 
radius $r_{\mathrm{cc}}+0.6 H_{\mathrm{P}}$ is chemically homogeneous. The misleading point is that 0.6 may be considered as a small number, but, $H_{\mathrm{P}}$ is not. If things were so easy, in other words, if there was a single overshooting distance for a given stellar mass (or, in general, for all masses), then, after many applications to stellar systems, we should already found the proper value(s). Therefore, the problems we are facing are much more complicated and change star by star.

The seismic investigations of Aerts et al. (2003) and Dupret et al. (2004) show that the role of overshooting is at least exaggerated: they find the overshooting distance as $0.1 H_{\mathrm{P}}$. Furthermore, these seismic studies rule out solid-body rotation: the inner regions rotate faster than the outer regions.

\section{Differential rotation}

We should remember that differential rotation is accompanied by contraction due to gravity, provided that the angular momentum transfer in the stellar interior conditions is not a sudden process. In this respect, the most suitable stars, to determine the effect of differential rotation on the stellar structure, are the stars very close to either the zero-age-main-sequence (ZAMS) or the terminal-age-main-sequence (TAMS). While the primary component of EK Cep is a good example of a ZAMS star, $\theta^{2}$ Tau A is a post-MS star, burning its hydrogen in the shell surrounding the He core.

Here we discuss the calibration method of a differentially rotating model for a given chemical composition. We assume that the model at the threshold of stability point, where it is completely convective (except for the photosphere), rotates like a solid-body, say, with an angular velocity $\Omega_{0}$. As a radiative region forms and as a result of contraction, differential rotation begins, the most rapidly rotating region is that which has contracted the most, namely the central region. If angular momentum is conserved (or equivalently not transfered instantaneously in radiative regions), the luminosity and the radius of a model with such rotational properties are functions of $\Omega_{0}$, for a given time.

As the synchronization for an early type star in a binary system starts from the surface regions (Zahn 1977, Goldreich \& Nicholson 1989), the rotation profile determined by contraction is changed in the outermost regions whose mass fraction is $M_{\mathrm{s}}$. If $M_{\mathrm{s}}$ is very small, then the luminosity is independent of $M_{\mathrm{S}}$ and the radius strongly depends on $M_{\mathrm{s}}$. Otherwise, both the luminosity and the radius are very sensitive functions of $M_{\mathrm{s}}$, depending upon how different are the rotation rates of the outer and the inner regions.

Since we have no information on the angular momentum flux throughout the stellar interior, to make the rotational velocity of the model at a given time agree with the observed (synchronized) velocity we directly modify the angular momentum of the outer region with $M_{\mathrm{s}}$. To avoid the transitive course of a model reorganizing itself, it is better to make this modification in the pre-main-sequence (PMS) phase as soon as mass fraction of the receding convective envelope is less than $M_{\mathrm{s}}$. Once, we determine the numerical derivatives of $L$ and $R$ of such a model, then, it is straightforward to calibrate it at any time to the observed values.

\section{Double-lined eclipsing binaries}

\subsection{EK Cep}

The fundamental properties of the components of EK Cep are well known (Andersen 1991): $M_{\mathrm{A}}=2.024 \pm 0.023 M_{\odot}, R_{\mathrm{A}}=1.579 \pm 0.007 R_{\odot}, \log \left(L_{\mathrm{A}} / L_{\odot}\right)=1.17 \pm 0.04$; $M_{\mathrm{B}}=1.121 \pm 0.012 M_{\odot}, \quad R_{\mathrm{B}}=1.315 \pm 0.006 \mathrm{R}_{\odot}$, and $\log \left(L_{\mathrm{B}} / L_{\odot}\right)=0.21 \pm 0.06$.

The most striking feature in this system is, which directs us toward differentially rotating models, that the ratio of the observed luminosities $\left(L_{\mathrm{A}} / L_{\mathrm{B}}\right)$ of the components 
is smaller than the ratio found from the models (for example with solar compositions), in both the PMS and the MS phases (see Figure 2 in Ylldiz (2003)). This is the case also for the ratio of the radii. These differences between the theoretical and the observational results cannot be removed by an interplay between hydrogen $(X)$ and heavy element abundances $(Z)$, and imply some more radical difference between the structures of the components than expected within the standard theory of stellar evolution (see also Marques et al. (2004)).

Adopting the most probable case, that is, the secondary star is at the point where its luminosity is the maximum near the MS while the primary star is exactly at the ZAMS point, three equations (one for coevalness of the components and two for radius and luminosity of the secondary) can be written down with three unknowns $(X, Z$ and the mixing-length parameter for the secondary component; see Yıldız (2003)). The solution of the three equations yields the following values (SET E in Yildız 2003):

$$
X=0.614, \quad Z=0.0395, \quad \alpha=1.30 .
$$

Nonrotating models with these values are in very good agreement with the observational results for the secondary star and the apsidal motion of the system, but are not in agreement with the observed primary star: the model expectations for the primary are brighter and greater than the observed values. These discrepancies can be eliminated by assuming a rapidly rotating core for this star which is a natural result of contraction plus the synchronization process due to tidal interactions (Zahn 1977).

The agreement between the theoretical and the observational results is obtained if the central regions of the rotating model of EK Cep A with SET E rotate 65 times faster than the surface, and the outer $48 \%$ of the star's mass rotates synchronously (surface equatorial velocity $=22 \mathrm{~km} \mathrm{~s}^{-1}$ ).

Indeed, the rotation profile we found depends on the chemical composition (or, on our assumption about the position of EK Cep A relative to the ZAMS point). If the solar metallicity is assumed, then the core rotates 29 times faster than the surface and the synchronized outer mass is $10 \%$ of the total mass.

These two rotating models of EK Cep A (with SET E and B) have the same luminosity and radius (at different times; $26 \mathrm{My}$ for SET E and 28.5 My for SET B), but the apsidal advance rates (AAR) computed from these models, using also the corresponding model of EK Cep B, are quite (but not exhaustively) different. While the AAR computed from the models with SET E is in perfect agreement with the observed AAR, the corresponding AAR for SET B is very close to the lower limit of the observed value. So, it is better to study a system which has more accurate data for the apsidal motion.

\subsection{PV Cas}

The fundamental properties of the components of the PV Cas binary system (Andersen 1991; Barembaum \& Etzel 1995) are: $M_{\mathrm{A}}=2.82 \pm 0.05 M_{\odot}, R_{\mathrm{A}}=2.297 \pm 0.021 R_{\odot}$, $L_{\mathrm{A}} / L_{\odot}=51.24 \pm 5 ; M_{\mathrm{B}}=2.76 \pm 0.06 M_{\odot}, R_{\mathrm{B}}=2.256 \pm 0.016 \mathrm{R}_{\odot}$, and $\log \left(L_{\mathrm{B}} / L_{\odot}\right)=$ $49.17 \pm 5$. The uncertainty in luminosity is computed from the uncertainties in radius and temperature, assuming the uncertainty of the latter as $200 \mathrm{~K}$.

This DLEB system is an excellent one in two respects: 1) It has a very short apsidal motion period $(91 \pm 2 \mathrm{y})$. and 2) Its Ap-like variation is confirmed in its light curve. It is shown in Ylldiz (2004) that there is no solution with nonrotating models and models rotating like a solid-body, in the sense that the models should give both the observed properties of the component stars and AAR of the system at the same time. As in the case of EK Cep, interplay between $X$ and $Z$ does not alter the case. Furthermore, overshooting, which is effective for the evolved models, worsens the situation. 


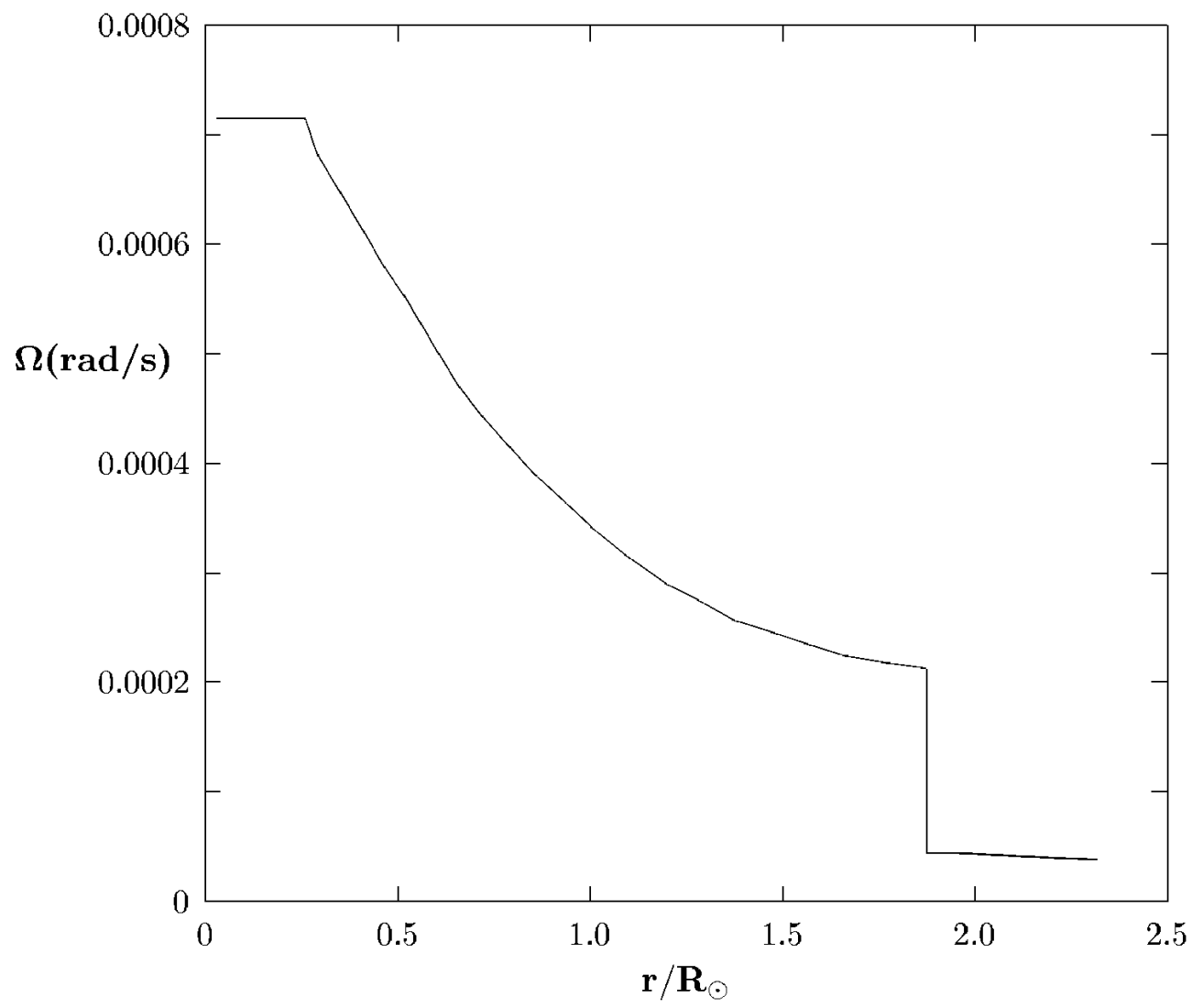

Figure 1. Internal rotation of model for PV Cas A as a function of radius. This model gives also the observed AAR.

Since the components of the binary system are almost identical $\left(M_{\mathrm{A}}=2.82 \pm 0.05 M_{\odot}\right.$, $M_{\mathrm{B}}=2.76 \pm 0.06 M_{\odot}$ ), we have no chance to find the chemical composition and the age of the system from model computations. Therefore, initially, a solar composition is assumed (for simplicity we also assume that the two components are identical = PV Cas A).

For the age $t=2.34 \times 10^{8} \mathrm{y}$, for example, after several tries we find that a model with $\Omega_{\mathrm{o}}=7.810^{-8} \mathrm{rad} \mathrm{s}^{-1}\left(\Omega_{\mathrm{c}}=8.510^{-4} \mathrm{rad} \mathrm{s}^{-1}\right)$ and $M_{\mathrm{s}}=0.016$ fits the observed luminosity, radius and observed rotational (=synchronized) velocity $\left(v_{\text {eq }}=65 \mathrm{~km} \mathrm{~s}^{-1}\right)$. This model does not gives the observed AAR at the corresponding time. The computed AAR is $23 \%$ greater than the observed value. Therefore, the amount of synchronized mass $M_{\mathrm{s}}$ should be decreased to reduce the theoretical AAR. Thus the solution we are seeking is at another time: At $t=1.4 \times 10^{8}$, the model with $\Omega_{\mathrm{o}}=6.710^{-8} \mathrm{rad} \mathrm{s}^{-1}$ $\left(\Omega_{\mathrm{c}}=7.110^{-4} \mathrm{rad} \mathrm{s}^{-1}\right)$ and $M_{\mathrm{s}}=0.000275$ is in perfect agreement with observations, including AAR. The angular velocity of this model is plotted in Figure 1 as a function of radius in solar units. The rotation rate is constant in the convective core and gradually decreases up to the base of the synchronized region $\left(\mathrm{r}=1.85 R_{\odot}\right)$ at which a sudden change occurs. This discontinuity is due to our simplification and there would be a smooth transition if the angular momentum transfer was computable in the physical conditions of stellar interiors.

If there is really such a sharp change in the rotational velocities of some stars, then it may somehow be confirmed by observations. The difference between effective 


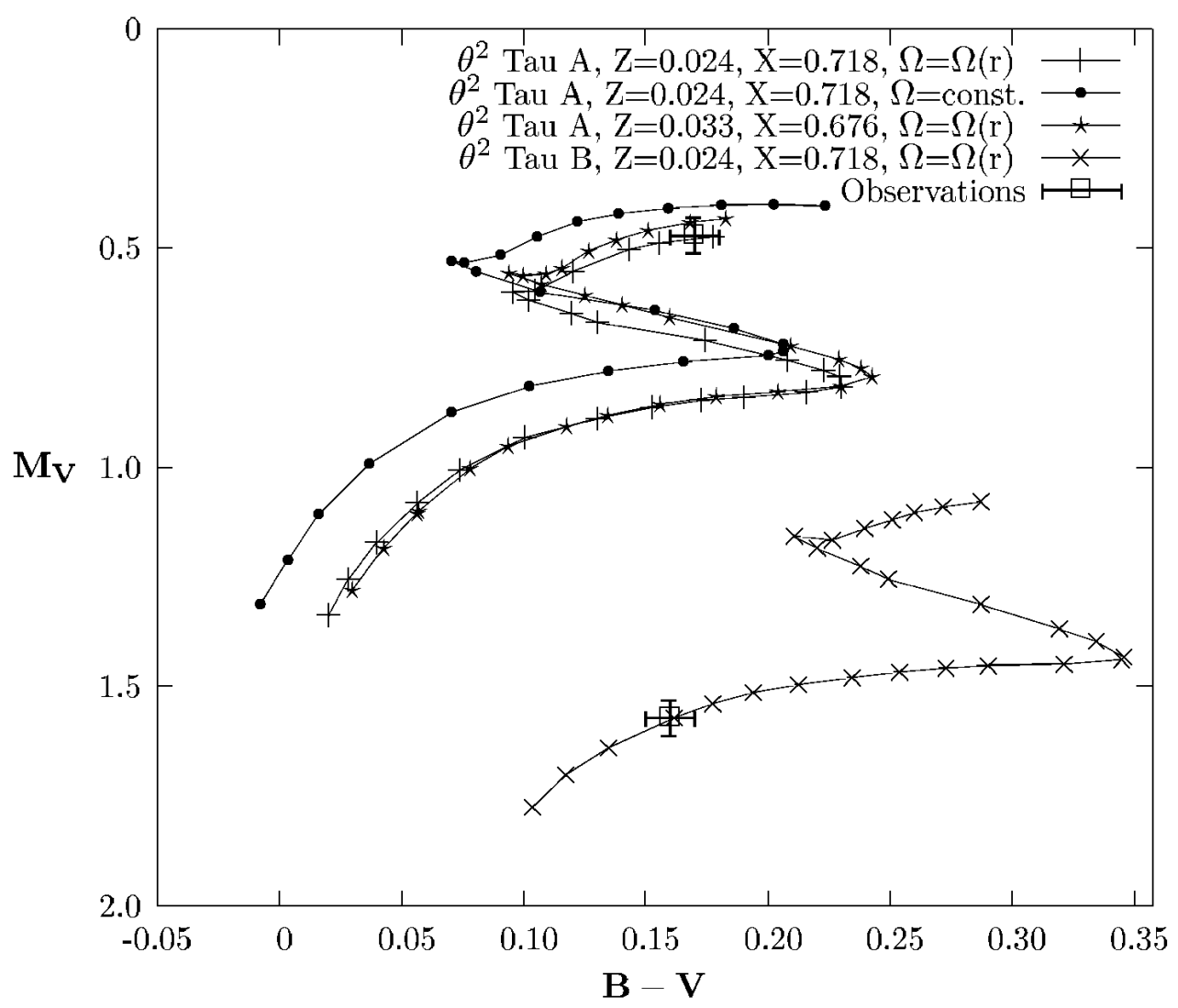

Figure 2. Color-magnitude diagram for $\theta^{2}$ Tau A and B.

temperatures of the magnetic Ap stars and normal stars found by Hubrig et al. (2000) may be treated in this context, since the same amount of difference exists also between the effective temperatures of non-rotating models and models with such differential rotation (see Yıldız 2005).

\section{The visual binaries $\theta^{2}$ Tau and $\phi 342$ in the Hyades open cluster}

Although the masses of the component stars in $\theta^{2}$ Tau found from the observations $\left(M_{\mathrm{A}}=2.42 \pm 0.30, M_{\mathrm{B}}=2.11 \pm 0.17\right)$ are not as accurate as for the DLEBs discussed in the previous section, we can extract information about the evolutionary phase and the structure of these stars, by considering them together with the components of $\phi 342$. While the calibration of latter gives $X$, for a given $Z$, we get the age of the cluster from calibration of $\theta^{2}$ Tau A. However, the age, for a given chemical composition, depends on the rotational properties assigned. Therefore, regarding the observated rotational velocity of $\theta^{2}$ Tau A ( $v_{\text {eq }} \sim 100 \mathrm{~km} \mathrm{~s}^{-1}$, Torres et al. 1997a), we consider the rotational properties of the inner regions for two typical cases: 1) solid-body rotation, and 2) differential rotation as determined by contraction (no synchronization is assumed since the system is very wide).

In the latter case, for $Z=0.024$, for example, $X=0.718$ and $t=721 \mathrm{My}$. In Figure 2, the evolutionary tracks of models $\theta^{2}$ Tau A $(+)$ and $\mathrm{B}(\times)$ with this chemical composition 
are plotted in the HRD. Both of the models are in very good agreement with the observed positions of the stars, but the agreement for $\theta^{2}$ Tau B is obtained at a different time $\left(t=440 \mathrm{My}\right.$; see below). On the other hand, the model of $\theta^{2}$ Tau A with solid-body rotation (filled circle) is not in agreement with the observation. However, it is not so easy to rule out solid-body rotation in such an analysis by regarding the superiority of the differentially rotating model since the uncertainty in mass is not sufficiently small $\left(M_{\mathrm{A}}=2.42 \pm 0.30\right)$. For comparison, another differentially rotating model for $Z=0.033$, for which $X=0.676$ and $t=671 \mathrm{My}$, is also plotted in Figure $2(\star)$. It is also in good agreement with the observed values, but, not as good as the model with $Z=0.024$.

As emphasized above both the differentially rotating models of $\theta^{2}$ Tau A and B with $Z=0.024$ are in agreement with the observations, but not at the same time. Although the observed rotational velocity of the secondary star is highly uncertain $(v \sin i=90-$ $170 \mathrm{~km} \mathrm{~s}^{-1}$; Torres et al. 1997a), it seems that maybe its rotation profile is somewhat different from that determined by contraction alone.

All the models of $\theta^{2}$ Tau A are compatible with the subgiant phase in which hydrogen is burnt in the shell surrounding the He core. If $\theta^{2}$ Tau A was an isolated star, we could neither be sure about its evolutionary aspect nor discuss its rotational properties by modelling.

\section{Conclusion}

The double-lined (eclipsing or visual) binaries, as unique sources of data for stellar mass, are essential systems for the determination of the physical properties and the evolutionary aspects of stars. We can assess the effect of internal rotation on the observable properties of the models for the components of these binaries, and then remove the discrepancy between the theoretical and observational results. In this respect, EK Cep, PV Cas and $\theta^{2}$ Tau are very fruitful systems. To make the properties of differentially rotating models agree with the all available observational constraints, the internal rotational properties of components stars follow:

1) EK Cep A, a ZAMS star, has a very rapidly rotating core: For $Z=0.04$, the core rotates 65 times faster than the surface and mass fraction of the synchronized outer mass is $48 \%$. For $Z=0.02$, the ratio of core rotation rate to that of the surface is 29 and the mass fraction is $10 \%$. While the models for EK Cep A with the two different chemical compositions have the same observable properties $(L$ and $R$ ), the theoretical AAR based on the models with a metal-rich composition is in better agreement than the models with solar metallicity. However, the latter is close to the lower limit of (but, still in) the observed range.

2) $\theta^{2}$ Tau A, a subgiant near to the TAMS, may have a much steeper differential rotation. While properties of its model with differential rotation as determined by contraction are in very good agreement with its observed position in the HRD, the track of model with solid-body rotation never touches the observational box. But, for a definite conclusion, its mass should be measured more accurately.

3) PV Cas A \& B seem to be slowly rotating stars, but the slow rotation is limited to a shallow region beneath the surface. For a solar composition, while only $0.03 \%$ of the outermost mass is synchronized, the rest is rotating very rapidly (see Figure 1). The confirmation of Ap-like variation in the light-curve of the system (Barembaum \& Etzel 1995) leads us to seek a correlation between the chemical peculiarity and the internal rotation. Furthermore, the temperature difference found between the magnetic Ap stars and normal stars exists also between NR models and models with such differential rotation. 


\section{Acknowledgements}

I thank Jean-Paul Zahn for his very stimulating discussions and suggestions, and Hiromoto Shibahashi for fruitful discussions during the symposium. Belinda Kalomeni is acknowledged for reading this manuscript.

\section{References}

Aerts, C., Thoul, A., Daszynska, J., et al. 2003, Science 300, 1926

Andersen, J. 1991, A\&AR 3, 91

Barembaum, M.J., \& Etzel, P.B. 1995, AJ 109, 2680

Claret, A. \& Gimenez, A. 1993, A\& A 277, 487

Dupret, M.-A., Thoul, A., Scuflaire, R., et al. 2004, A\&A 415, 251

Goldreich, P. \& Nicholson, P.D. 1989, ApJ 342, 1075

Guinan, E.F., Ribas, I., Fitzpatrick, E.L., et al. 2000, ApJ 544, 409

Harmanec, P. 1988, BAICz 39, 329

Hubrig, S., North, P., \& Mathys, G. 2000, ApJ 539, 352

Lacy, C.H.S., Torres, G., Claret, A. \& Sabby, J.A. 2003, AJ 126, 1905

Lastennet, E., \& Valls-Gabaud, D. 2002, A\& $A$ 396, 551

Lebreton, Y., Fernandes, J., \& Lejeune, T. 2001, A\&A 374, 540

Marques, J.P., Fernandes, J., \& Monteiro, M.J.P.F.G. 1997, A $\& A$ 239, 245

Pols, O.R., Tout, C.A., Schroder, K-P., Eggleton, P.P., Manners, J. 1997, MNRAS 289, 869

Popper, D. 1980, ARAESA 18, 115

Roxburgh, I.W. 1992, A\&SA 266, 291

Torres, G., Stefanik, R. P., \& Latham, D. W. 1997a, ApJ 485, 167

Torres, G., Stefanik, R. P., \& Latham, D. W. 1997b, ApJ 479, 268

Yıldız, M. 2003, A\&A 409, 689

Yıldız, M. 2004, A\&SA, submitted

Ylldı, M. 2005, These Proceedings, 89

Young, P.A., Mamajek, E.E., Arnett, D., \& Liebert, J. 2001,ApJ 556, 230

Zahn, J.-P. 1977, A\&A 57, 383

\section{Discussion}

Moss: It is a basic idea that nonaxisymmetric fields, as seen in Ap stars (especially the more rapid rotators), and strong differential rotation are incompatible - either the differential rotation destroys the nonaxisymmetric field, or the differential rotation is strongly reduced by the magnetic field.

How are your ideas compatible with this? How do you explain this problem in the context of your ideas?

YILDIZ: A very steep differential in depth is found near the surface for the components of PV Cas. But, in our models for the primary, the outermost $0.03 \%$ of the total mass ( $19 \%$ by radius) is rotating slowly. Maybe, this is the part where diffusion works and the magnetic structure has the same character as that of the observed surface. 